\title{
Structure and Evolution of Neurexophilin
}

\author{
Alexander G. Petrenko, ${ }^{1,2}$ Beate Ullrich, ${ }^{1}$ Markus Missler, ${ }^{1}$ Valery Krasnoperov, ${ }^{2}$ Thomas W. Rosahl, ${ }^{1}$ and \\ Thomas C. Südhof ${ }^{1}$ \\ ${ }^{1}$ Howard Hughes Medical Institute and Department of Molecular Genetics, The University of Texas Southwestern Medical \\ School, Dallas, Texas 75235, and 2Department of Environmental Medicine, New York University Medical Center, New \\ York, New York 10016
}

\begin{abstract}
Using affinity chromatography on immobilized $\alpha$-latrotoxin, we have purified a novel $29 \mathrm{kDa}$ protein, neurexophilin, in a complex with neurexin $l \alpha$. Cloning revealed that rat and bovine neurexophilins are composed of $\mathrm{N}$-terminal signal peptides, nonconserved $\mathrm{N}$-terminal domains (20\% identity over 80 residues), and highly homologous C-terminal sequences $(85 \%$ identity over 169 residues). Analysis of genomic clones from mice identified two distinct neurexophilin genes, one of which is more homologous to rat neurexophilin and the other to bovine neurexophilin. The first neurexophilin gene is expressed abundantly in adult rat and mouse brain, whereas no mRNA corresponding to the second gene was detected in rodents despite its abundant expression in bovine brain, suggesting that rodents and cattle primarily express distinct neurexophilin genes.
\end{abstract}

$\alpha$-Latrotoxin, a component of black widow spider venom, is a potent presynaptic neurotoxin (Frontali et al., 1976; Rosenthal and Meldolesi, 1989). Acute administration of $\alpha$-latrotoxin causes massive neurotransmitter release; chronic exposure results in cell membrane damage and synaptic degeneration. An understanding of the molecular mechanisms of $\alpha$-latrotoxin toxicity is beginning to emerge. The toxin binds to high-affinity receptors that are localized in the presynaptic plasma membrane of the nerve terminal (Tzeng and Siekevitz, 1979; Valtorta et al., 1984). Binding of toxin results in $\mathrm{Ca}^{2+}$ influx into nerve terminals, activation of synaptic vesicle exocytosis, and ATP depletion (Grasso et al., 1980; McMahon et al., 1990). Interestingly, $\alpha$-latrotoxin triggers synaptic vesicle exocytosis even in the absence of extracellular $\mathrm{Ca}^{2+}$, suggesting that the toxin stimulates $\mathrm{Ca}^{2+}$-independent vesicle exocytosis (Misler and Hurlbut, 1979).

A protein complex that binds $\alpha$-latrotoxin with high affinity was purified from bovine and rat brain and shown to consist of a set of high molecular weight proteins of $160-220 \mathrm{kDa}$ and a low molecular weight protein of $29 \mathrm{kDa}$ (Petrenko et al., 1990, 1993). The high molecular weight proteins represent splice variants of neurexin I $\alpha$ (Ushkaryov et al., 1992), and recombinant neurexin $\mathrm{I} \alpha$ binds $\alpha$-latrotoxin with high affinity (Davletov et al., 1995). The

\footnotetext{
Received Feb. 22, 1996; revised April 19, 1996; accepted April 24, 1996.

This research was supported by National Institutes of Health Grants MH52804 (T.C.S.) and NS34937 (A.G.P.), and by fellowship grants from the Deutsche Forschungsgemeinschaft to B.U., M.M., and T.W.R. We thank I. Leznicki, A. Roth, H.

Tripoli, and E. Borowicz for excellent technical assistance; Drs. M. S. Brown and J. L. Goldstein for critical advice; and Drs. J. Dixon and D. W. Russell for the gift of expression vectors and cDNA libraries.

Correspondence should be addressed to Thomas C. Südhof, Howard Hughes Medical Institute, Room Y5.322, 5323 Harry Hines Boulevard, Dallas TX 75235.

Copyright (C) 1996 Society for Neuroscience 0270-6474/96/164360-10\$05.00/0
}

RNA blots and in situ hybridizations revealed that neurexophilin is expressed in adult rat brain at high levels only in a scattered subpopulation of neurons that probably represent inhibitory interneurons; by contrast, neurexins are expressed in all neurons. Neurexophilin contains a signal sequence and is $\mathrm{N}$-glycosylated at multiple sites, suggesting that it is secreted and binds to the extracellular domain of neurexin $I \alpha$. This hypothesis was confirmed by binding recombinant neurexophilin to the extracellular domains of neurexin $1 \alpha$. Together our data suggest that neurexophilin constitutes a secreted glycoprotein that is synthesized in a subclass of neurons and may be a ligand for neurexins.

Key words: neurexins; $\alpha$-latrotoxin; synapse; gene duplication; neuron-specific receptor; inhibitory interneurons identity and characteristics of the low molecular weight protein, however, remain unknown.

Neurexins are membrane proteins with the characteristics of cell-surface receptors. They are encoded by at least three genes, each of which has two independent promoters directing transcription of longer $\alpha$-neurexins and shorter $\beta$-neurexins. The neurexin transcripts are subject to extensive alternative splicing (Geppert et al., 1992; Ushkaryov et al., 1992, 1994; Ushkaryov and Südhof, 1993). This probably results in the synthesis of $>1000$ neurexin isoforms that are expressed differentially in subpopulations of neurons in the brain (Ullrich et al., 1995). The domain structure of the neurexins suggests a receptor function, possibly in cell adhesion. In support of this hypothesis, a family of neuronal cell surface proteins named neuroligins, which bind to $\beta$-neurexins in a splice variant-specific manner, was described recently (Ichtchenko et al., 1995, 1996). It seems likely that additional ligands for neurexins that may also be splice-site specific will be discovered. On the intracellular side, neurexins contain a short cytoplasmic tail that interacts with the synaptic vesicle protein synaptotagmin (Petrenko et al., 1991; Hata et al., 1993) and with a novel protein called CASK that contains CaM kinase- and dlg-like sequences (Hata et al., 1996).

We have now studied the structure, properties, and expression of the low molecular weight protein that is purified on immobilized $\alpha$-latrotoxin. We propose to name this protein neurexophilin because of its strong binding to neurexin $\mathrm{I} \alpha$. Our data reveal that neurexophilin is a secreted cysteine-rich glycoprotein. Neurexophilin is expressed in a brain-specific manner in a subset of neurons that are scattered throughout the nervous system. Together our data raise the possibility that neurexophilin represents a novel ligand for $\alpha$-neurexins. 


\section{MATERIALS AND METHODS}

Cloning and sequencing of cDNAs and genomic clones encoding neurexophilins. A PCR-based cDNA cloning strategy was used to take advantage of the single short peptide sequence (sequence: VVEFEVSPQSTLETK) obtained previously from purified neurexophilin (Petrenko et al., 1993). PCRs using primers $\mathrm{A}$ and $\mathrm{B}$ or $\mathrm{C}$ (sequences: $\mathrm{A}=$ GGCTGCAGTNGTNGA[G,A]TT[C,T] GA; B = GGTCTAGAA[A,G,T]CA[G,A]AGNT CNCA; and $\mathrm{C}=$ GGTCTAGAANCA[A,G]AG[A,G]TTNCA; $n=$ all nucleotides; other redundant nucleotide combinations are shown in brackets) were performed on single-stranded bovine brain cDNA as template, as described by Ushkaryov and Südhof (1993). PCR products of approximately the right size were subcloned in M13 vectors and analyzed by sequencing. One M13 clone with the correct sequence was identified and used to screen cDNA libraries (Sambrook et al., 1989; Südhof, 1990). Mouse genomic clones were isolated from different $\lambda$ libraries using the rat cDNA as a probe and mapped and sequenced, essentially as described (Südhof, 1990). All DNA sequencing was performed on M13 subclones using the dideoxy nucleotide-chain termination method (Sanger et al., 1977) with fluorescently labeled primers, Taq DNA polymerase, and an ABI373A DNA sequencer for analysis. Sequences were analyzed on a PC using Intelligenetics software and submitted to Genbank (accession numbers L27867, L29868, U56650, and U56651).

$R N A$ preparation and blotting. Total RNA isolated from different rat and bovine tissues by guanidinium isothiocyanate extraction was analyzed by blotting, using uniformly ${ }^{32} \mathrm{P}$-labeled probes as described (Ushkaryov et al., 1992). To control for RNA loads, blots were rehybridized with a cyclophilin probe.

In situ hybridizations were performed on rat brain sections using ${ }^{35} \mathrm{~S}$-labeled oligonucleotides corresponding to rat and bovine neurexophilins, essentially as described (Gerfen et al., 1992; Ullrich et al., 1995). Two controls were performed to verify specificity of labeling. First, hybridizations were performed in a 50- and 100-fold excess of unlabeled oligonucleotide to determine which signal could be competed away and was therefore specific. Second, two antisense oligonucleotides from different regions of the mRNAs were used for the experiments to ensure that the signal that was observed corresponded to the mRNA being studied. Neurexophilin 1 oligonucleotides used for in situ hybridizations were T964 and T1065 [sequences (redundant positions shown in brackets): CCAGGACAC[A,G]TGACT[C,T]TG[A,G]GTCTGCTCCT GGTA[G,A]CAG and AATCTGTGCCATTTTCTTTACCACGGAAAGTCTGTGAGAGGA] and T1066 (CTGGAGA CTGTTTAACAAACAGGCGCAGAGGGTTGATGATCC) for neurexophilin 2.

Antibodies. The antibodies used for the current study were raised against the following synthetic peptides coupled to keyhole limpet hemocyanin (Johnston et al., 1989; Petrenko et al., 1993): (1) a synthetic peptide containing residues $261-271$ of rat neurexophilin (the $\mathrm{C}$ terminus), which in bovine neurexophilin exhibits three amino acid substitutions (F508); (2) a peptide containing residues 167-179 of bovine neurexophilin of which only five residues are conserved in rat neurexophilin (A550); (3) a peptide from the C terminus of neurexin II that reacts with all neurexins (A473) (Ushkaryov et al., 1992). Specificities of the antibodies were assessed by competition studies, with the respective synthetic peptides used for immunizations.

Expression of neurexophilins and IgG-fusion proteins by transfection in COS cells. Expression vectors encoding full-length bovine and rat neurexophilins (pCMV 1 and pCMV $\Delta 2$, respectively) were constructed by subcloning the cDNA clones into the appropriate pCMV vectors. The expression construct encoding the $\mathrm{N}$-terminal truncated rat neurexophilin preceded by the signal sequence (pCMV 33$)$ was obtained by deleting the coding sequence for the cleaved pro-peptide portion by PCR-based site-directed mutagenesis. IgG-neurexin I $\alpha$ and IgG-control proteins were produced in COS cells by transfection of previously described constructs (Ichtchenko et al., 1995). DNA transfections into COS cells were performed as described (Gorman, 1985), using DEAE-dextran.

$\alpha$-Latrotoxin affinity chromatography was performed using homogenates from frozen bovine and rat brains, as described (Petrenko et al., 1993). The protein components of the preparations were characterized by SDS-PAGE followed by Coomassie blue staining or by immunoblotting with antibodies to neurexins and neurexophilin.

Sucrose gradient and gel filtration experiments. The nature of the binding of neurexophilin to neurexins purified on immobilized $\alpha$-latrotoxin was studied by sucrose gradient centrifugations in the presence of different concentrations of $\mathrm{NaCl}$ and different detergents and by gel filtration on a Sephacryl S-300 column $(7 \times 137 \mathrm{~mm})$ equilibrated with either $6 \mathrm{M}$ urea,
$75 \mathrm{~mm}$ Tris- $\mathrm{HCl}, \mathrm{pH} 7.4$, or with $7 \mathrm{~m}$ guanidinium- $\mathrm{HCl}, \mathrm{pH}$ 1.7. The neurexin/neurexophilin samples were applied to and eluted from the column with the respective buffers at a flow rate of $7.5 \mathrm{ml} / \mathrm{hr}$. Fractions $(0.1 \mathrm{ml})$ were analyzed by electrophoresis and immunoblotting with anti-neurexophilin and anti-neurexin antibodies.

Binding of recombinant neurexin I $\alpha$ to neurexophilin. Recombinant neurexin $\mathrm{I} \alpha$-IgG and control IgG-fusion proteins were immobilized on protein A-agarose $(20 \mu \mathrm{l} / 1 \mathrm{ml}$ culture medium $)$ from the medium of transfected COS cells after the addition of $40 \mathrm{~mm}$ Tris- $\mathrm{HCl}, \mathrm{pH}$ 8.0. The portion of the rat neurexophilin cDNA encoding residues 111-271 was subcloned into the $X b a \mathrm{I}$ site of pGEX-KG using PCR. Recombinant GST-fusion proteins were expressed and purified as described on glutathione-agarose (Smith and Johnson, 1988). Protein eluted from the glutathione agarose column was purified additionally by DEAESepharose for some experiments. The protein A-agarose beads containing recombinant $\mathrm{IgG}$-fusion proteins were washed twice with incubation buffer ( $40 \mathrm{~mm}$ Tris- $\mathrm{HCl}, \mathrm{pH}$ 8.0, $0.3 \mathrm{M} \mathrm{NaCl}, 1 \%$ Triton $\mathrm{X}-100,1 \%$ bovine serum albumin), preincubated in $80 \mu \mathrm{l}$ of this buffer for $2 \mathrm{hr}$, and then incubated $30 \mathrm{~min}$ after the addition of $\sim 1 \mu \mathrm{g}$ GST-neurexophilin added in $20 \mu \mathrm{l}$ of the same buffer containing $5 \mathrm{~mm}$ glutathione but lacking bovine serum albumin. After incubation, samples were centrifuged, and the beads were washed twice with $40 \mathrm{~mm}$ Tris- $\mathrm{HCl}, \mathrm{pH} 8.0,0.15 \mathrm{M} \mathrm{NaCl}$. Beads were resuspended in $40 \mu \mathrm{l}$ SDS-PAGE sample buffer, and $10 \mu \mathrm{l}$ of the bead fraction and the supernatants from the incubations were analyzed by SDS-PAGE and immunoblotting.

Deglycosylation of native and recombinant neurexophilin. Proteins purified by affinity chromatography on $\alpha$-latrotoxin $(30 \mu \mathrm{g} / \mathrm{ml}$ protein) were dialyzed against $50 \mathrm{~mm} \mathrm{Na}_{2} \mathrm{HPO}_{4}, \mathrm{pH} 7.4,0.1 \%$ Triton X-100, to remove $\mathrm{KCl}$, denatured with SDS $/ \beta$-mercaptoethanol, and treated with recombinant PNGase F (New England Biolabs, Beverly, MA) with control incubations in the absence of the enzyme. Transfected COS cells and their culture media were digested with PNGase F in the same manner. Samples were analyzed by SDS-PAGE and immunoblotting using antineurexophilin antibodies.

\section{RESULTS}

\section{Molecular cloning of rat and bovine neurexophilins}

Only a single short peptide sequence from bovine neurexophilin was obtained in previous studies (sequence: VVEFEVSPQSTLETK; Petrenko et al., 1993). This sequence contains two serine residues, making direct screening of cDNA libraries with redundant oligonucleotides difficult. Therefore we performed PCRs on single-stranded bovine brain cDNA templates using highly degenerate oligonucleotides encoding the $\mathrm{N}$ - and C-terminal five amino acids of this peptide (see Materials and Methods). PCR products of the expected size (58 bp) were cloned into M13 vectors and sequenced. One M13 clone had the correct sequence and was used as a probe for cDNA screening, resulting in the identification of partial bovine neurexophilin cDNA clones. These were then used as probes to isolate rat and bovine cDNA clones for neurexophilin that were full-length with respect to the coding region ( $\mathrm{p} \Delta 7$ and $\mathrm{p} \Delta 14)$.

The cDNA sequences were used to determine the amino acid sequences of rat and bovine neurexophilins (shown aligned with each other in Fig. $1 A$ ). Databank searches revealed no significant similarity to current entries, suggesting that the neurexophilins are novel proteins. Analyses of the rat and bovine sequences suggested that the primary translation product of neurexophilin has a multidomain structure (Fig. 1B). At the $\mathrm{N}$ terminus, rat and bovine neurexophilins contain a single hydrophobic sequence that has the characteristics of a signal peptide with putative cleavage sites after residues 21 (rat) or alternatively 20 or 22 (bovine) (von Heijne, 1987). After the putative signal sequence, an 80-residue sequence is observed in neurexophilins that exhibits little similarity between the rat and bovine sequences ( $20 \%$ identity) but ends in a conserved polybasic sequence (double-underlined in Fig. 1A). After this nonconserved region, rat and bovine neurexophilin contain a 
A

Figure 1. Structure of rat and bovine neurexophilins. $A$, Amino acid sequences of rat and bovine neurexophilins. Protein sequences were deduced from the cDNA nucleotide sequences (Genbank accession numbers L27867 and L29868). Sequences are identified on the left ( $R$, rat; $B$, bovine neurexophilin) and numbered on the right. Residues that are different between the rat and bovine sequences are marked by asterisks. The putative $\mathrm{N}$-terminal signal sequence is shown in bold typeface, and its predicted cleavage site is marked by an arrow. The basic tetrapeptide sequence (RTKR) at the boundary between variable and conserved domains is double-underlined and shown in bold typeface. Conserved cysteine residues are shaded, and the four $\mathrm{N}$-linked glycosylation consensus sequences are underlined. $B$, Domain structure of neurexophilin. Four domains are proposed: (1) a signal peptide at the $\mathrm{N}$ terminus; (2) a nonhomologous $\mathrm{N}$-terminal region that may constitute a propeptide; (3) a highly conserved middle segment that contains three $\mathrm{N}$-glycosylation consensus sequences (branched lines); and (4) a conserved C-terminal sequence characterized by six conserved cysteine residues (indicated by $C$ ).
$\mathrm{R}$

$\mathbf{B}$

$\mathbf{R}$

B

$\mathbf{R}$

R
B
M츤 M-RLRPLPLVVVPGLLQLLFCD SEKVVHATEGLDWEDKDATG----TLVGN-VV

츤 HSRII-NPLRLFVKQSPVPKPGHLAYADSMENFWDWLANITEVQEPLARTKRRPI 102

VKTGKFKKMFGWGDFHSNIKTVKLNLLITGKIVDHGNGTFSVYैFRHNSTGQGNVS 164 VKTGKFKKMFGWGDFHSNIKTVKLNLLITGKIVDHGNGTFSVYFRHNSTGLGNVS 157

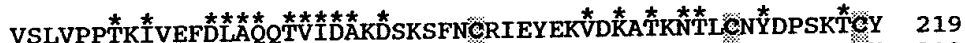
VSLVPPSKVVEFEVSPQSTLETKESKSFNCRIEYEKTDRAKKTALENFDPSKICY 212

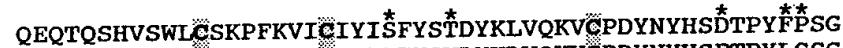
QEQTQSHVSWLESKPFKVIEIYIAFYSVDYKLVQKVCPDYNYHSETPYLSSG
271

264

B

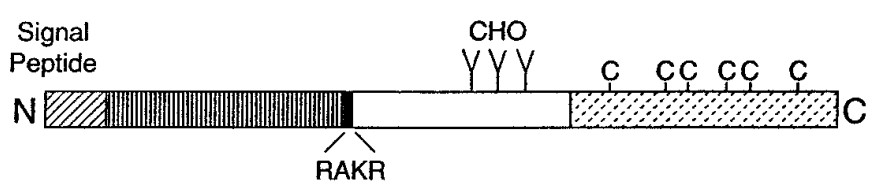

Variable Domains | Conserved Domains

169-residue sequence that is well conserved ( $85 \%$ identity). This sequence can be subdivided into an N-terminal half that exhibits only a single amino acid substitution between rat and bovine neurexophilin and contains three N-glycosylation sites, and a C-terminal half that is characterized by six conserved cysteine residues (shaded in Fig. 1A). The two conserved C-terminal domains are separated from each other by a short stretch of a nonconserved sequence (residues 171-188 in rat neurexophilin). Thus the domain model of neurexophilins suggests that they are composed of an $\mathrm{N}$-terminal signal peptide, an $\mathrm{N}$-terminal variable domain, and two $\mathrm{C}$-terminal conserved domains (Fig. 1B).

\section{Characterization of murine neurexophilin genes}

Rat and bovine neurexophilin exhibit no significant sequence homology with each other in their signal sequences and the following $\mathrm{N}$-terminal 87 residues and are only $85 \%$ identical over their C-terminal regions. This is an unusually low degree of similarity for the same proteins from different mammals, suggesting the possibility that the proteins we cloned may be isoforms instead of homologs. To investigate this possibility, we screened a murine genomic library by low-stringency hybridization with coding region probes from neurexophilins. Two classes of clones were isolated that correspond to two different neurexophilin genes (Fig. 2). In both classes of genomic clones, a single large exon encoding neurexophilin was identified that initiates at the end of the signal sequence and contains all of the remaining coding sequence (Fig. $3 A, B)$. The exon is preceded by a typical intron acceptor site (underlined in Fig. $3 A, B$ ) and as far as sequenced also contains the complete $3^{\prime}$ untranslated region of the neurexophilin mRNA. Only the first 18 and 17 amino acids encoded by the rat and bovine cDNAs are missing in the exon, suggesting that the complete coding sequence for neurexophilin is contained in two exons, one encoding the signal peptide and the second encoding the remaining parts of the protein. The first exon was not identified in the genomic clones isolated, indicating that the intron in the gene is rather large.

Comparison of the protein sequences predicted from the genomic clones with those of rat and bovine neurexophilins revealed that one of the genes is more similar to rat neurexophilin and is referred to as the neurexophilin 1 gene. By contrast, the second gene, referred to as the neurexophilin 2 gene, is more similar to bovine neurexophilin (Fig. $3 C$ ). In the N-terminal nonconserved domain, murine neurexophilin 1 is almost identical

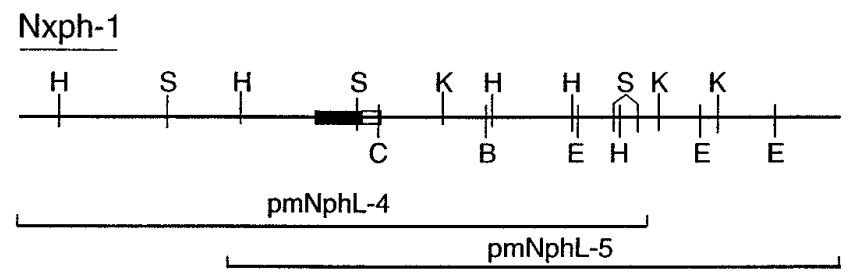

Nxph-2

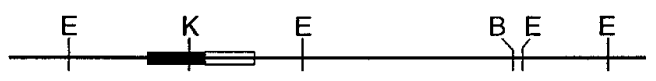

pm L-9-1

$1 \mathrm{~kb}$

Figure 2. Partial structure of murine neurexophilin genes. The diagram depicts restriction maps of the murine neurexophilin 1 (Nxph-1) and neurexophilin 2 (Nxph-2) genes as deduced from the genomic clones identified below the maps. Restriction enzyme cleavage sites are indicated by letters (B, BstBI; C, ClaI; E, EcoRV; H, HindIII; K, KpnI; S, SpeI). The locations of exons are marked by boxes; closed boxes indicate coding and open boxes indicate $3^{\prime}$ untranslated regions. The scale of the drawing is given in the lower right corner. 
A

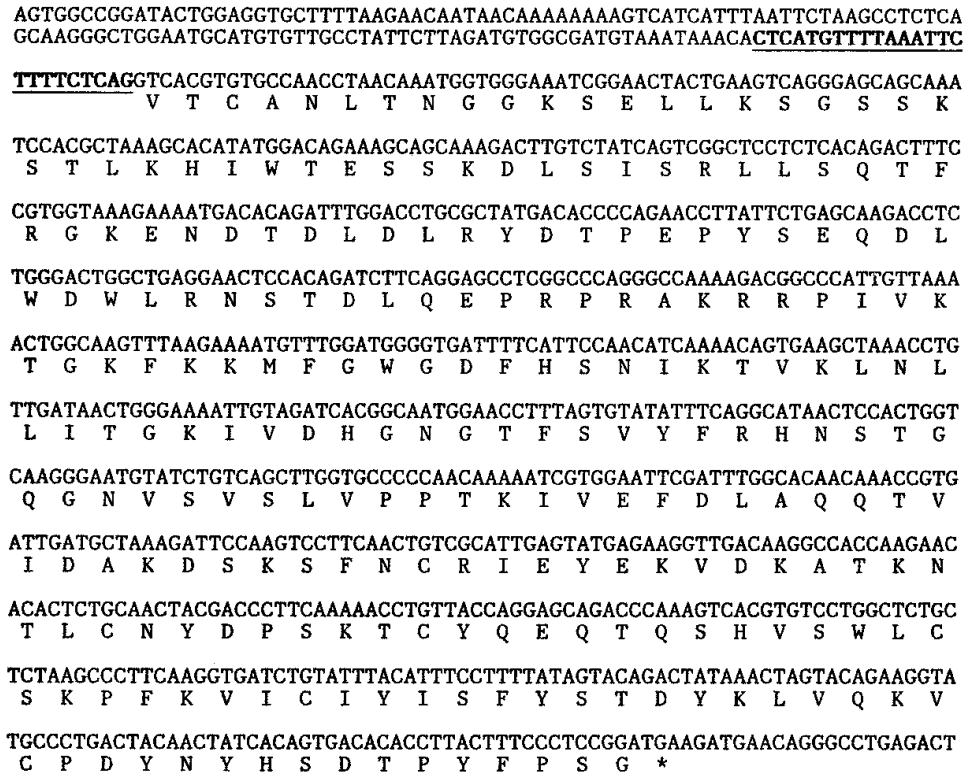
GCAAGGGCTGGAATGCATGTGTTGCCTATTCTTAGATGTGGCGATGTAAATAAACACTCATGTTTTAAATTC TTTTCTCAGGTCACGTGTGCCAACCTAACAAATGGTGGGAAATCGGAACTACTGAAGTCAGGGAGCAGCAAA TCCACGCTAAAGCACATATGGACAGAAAGCAGCAAAGACTTGTCTATCAGTCGGCTCCTCTCACAGACTTTC $\begin{array}{llllllllllllllllllllllll}\mathbf{S} & \mathrm{T} & \mathrm{L} & \mathrm{K} & \mathrm{H} & \mathrm{I} & \mathrm{W} & \mathrm{T} & \mathrm{E} & \mathrm{S} & \mathrm{S} & \mathrm{K} & \mathrm{D} & \mathrm{L} & \mathrm{S} & \mathrm{I} & \mathrm{S} & \mathrm{R} & \mathrm{L} & \mathrm{L} & \mathbf{S} & \mathrm{Q} & \mathrm{T} & \mathrm{F}\end{array}$ CGTGGTAAAGAAAATGACACAGATTTGGACCTGCGCTATGACACCCCAGAACCTTATTCTGAGCAAGACCTC $\begin{array}{llllllllllllllllllllllll}R & G & K & E & N & D & T & D & L & D & L & R & Y & D & T & P & E & P & Y & S & E & Q & D & L\end{array}$ TGGGACTGGCTGAGGAACTCCACAGATCTTCAGGAGCCTCGGCCCAGGGCCAAAAGACGGCCCATTGTTAA

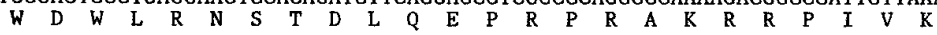

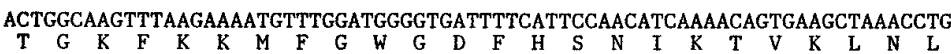
TTGATAACTGGGAAAATTGTAGATCACGGCAATGGAACCTTTAGTGTATATTTCAGGCATAACTCCACTGGT

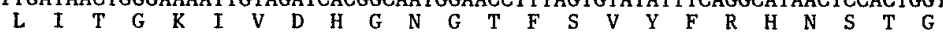

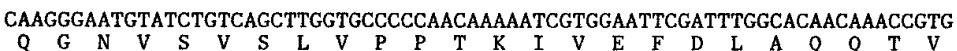
ATTGATGCTAAAGATTCCAAGTCCTTCAACTGTCGCATTGAGTATGAGAAGGTTGACAAGGCCACCAAGAAC

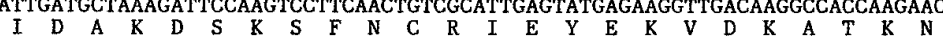
$\underset{T}{\text { ACACTCTGCAACTACGACCCTTCAAAAACCTGTTACCAGGAGCAGACCCAAAGTCACGTGTCCTGGCTCTGC }}$ TCTAAGCCCTTCAAGGTGATCTGTATTTACATTTCCTTTTATAGTACAGACTATAAACTAGTACAGAAGGTA $\begin{array}{llllllllllllllllllllllllllll}S & K & P & F & K & V & I & C & I & Y & I & S & F & Y & S & T & D & Y & K & L & V & Q & K & V\end{array}$ TGCCCTGACTACAACTATCACAGTGACACACCTTACTTTCCCTCCGGATGAAGATGAACAGGGCCTGAGACT $\begin{array}{lllllllllllllllll}\text { C } & \text { P } & \text { D } & Y & \text { N } & \text { Y } & \text { H } & \text { S } & \text { D } & \text { T } & \text { P } & \text { Y } & \text { F } & \text { P } & \text { S } & G & \text { G }\end{array}$

GAAGCCTGCGGAGTTAAAGGTCATAGGACAGGGCTGTCACCTTAAGGAAGAAGGTCACATCTGTTGCCTGGA ATGTGTCTACCTTGCTGCTCTTGTTGACTGGTGCACACATGCTAGTGGAAAACAATTGATGTCATTTCTGCA

B

Neurexophilin 2 Gene

TCATTCCCTTTTGCCTATTAACCTATGTCTGTATTCAAACCCTGTCCCAATGTGTGTGTGTGTGTATGTGAT ATGTACACATACTACACACATACTCTCTTTCTTCTTCTCCTACAGCTATTTTGTGACAGTGAAGAAGTGATA

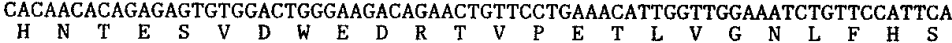
AGGATTACCAGTCCTCTGCGTCTGTTTGTTAAACAGCCTCCAGATCCAAAGCCATCATATGCAGACAACACG $\begin{array}{llllllllllllllllllllllllllll}R & I & T & S & P & L & R & L & F & V & K & Q & P & P & D & P & K & P & S & Y & A & D & N & T\end{array}$ AAAAACTTTTGGGATTGGCTGGCAAACATAACAGAAATTCAGGAGCAACTAGCAAGAACTAAAAGACGGCCA ATAGTGAAGACAGGAAAATTTAAAAAAATGTTTGGGTGGGGTGATTTCCACTCTAACATTAAAACTGTCAAA

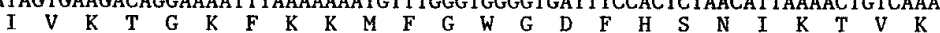
CTTAACTTGCTCATCACAGGAAAAATTGTTGATCATGGAAACGGAACATTCAGTGTTTATTTCCGACACAAT $\begin{array}{llllllllllllllllllllllllll}L & N & L & L & I & T & G & K & I & V & D & H & G & N & G & T & F & S & V & Y & F & R & H & N\end{array}$ TCAACAGGTCTGGGCAATGTTTCAGTGAGCTTGGTACCTCCCTCTAAAGTAGTAGAATTTGAAATATCCCCC

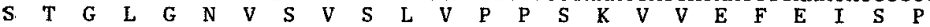
CAGTCTACCTTGGAGACTAAGGAATCCAAATCTTTTAACTGCCATATTGAGTATGAAAAAACAGATCGGGCA

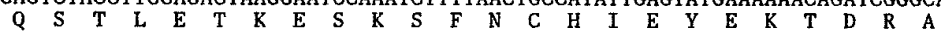
AAGAAGACCGCTTTGTGCAATTTCGACCCATCCAAAATCTGCTACCAGGAGCAGACTCAGAGTCATGTGTCC $\begin{array}{llllllllllllllllllllllll}K & K & T & A & L & C & N & F & D & P & S & K & I & C & Y & Q & E & Q & T & Q & S & H & V & S\end{array}$

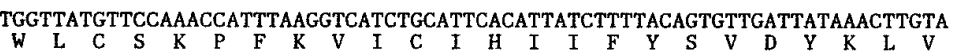

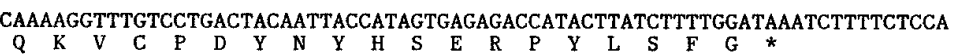
ATAGTAATAGAAATGAAATATTCATGTTACTTTACCTAGGAATGCTCATAAAACAGTGCCAGATTTCCATGG CAAAAAAATCCTTCTATTTCTGGCAGTGGACTGCAATTCCTTGTCCAGATTTCAAATTTTAAAAATATATTC

C

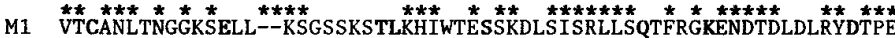
$R$ VTCANLTNGGKSELL--KSGNSKSTLKHIWTESSKDLSISRLLSQTFRGKENGTDLDLRYDTPE M2 LFCDSEEVIHNTESVDWEDRTVPETLVGNLFHSRITSPLRLFVKOPPDPKPS - - - - YAD- B LFCDSEKVVHATEGLDWEDKDATGTLVGNVVHSR I INPLRLFVKQSPVPKPG--HL-AYAD--

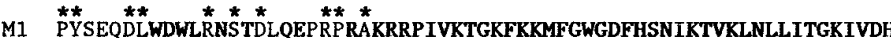
$R$ PYSEQDLWDWLRNSTDLQEPRPRAKRRPIVKTGKFKKMFGWGDFHSNIKTVKLNLLITGKIVDH 14 M2 --NTKNFWDWLANITEIOEOLARTKRRPIVKTGKFKKMFGWGDFHSNIKTVKLNLLITGKIVDH 117 B - SMENFWDHLANITEVQEPLARTKRRPIVKTGKFKKMFGWGDFHSNIKTVKLNLITGKIVDH

M1 GNGTFSVYFRHNSTGQGGNVSSLVPPTKIVEFDLAQQTVIDAKDSKSFNCRIEYEKVDKATKNT 190 $R$ GNGTFSVYFRHNSTGQGNVSVSLVPPTKIVEFDLAQQTVIDAKDSKSFNCRIEYEKVDKATKNT 208 M2 GNGTFSVYFRINSTGLGNVSVSIVPPSKVVTEEISPOSTLETKESKSFNCHIEYTKTDPAKKTA 181 B GNGTFSVYFRHNSTGLGNVSVSLVPPSKVVEFEVSPQSTLETKESKSFNCRIEYEKTDRAKKTA 201

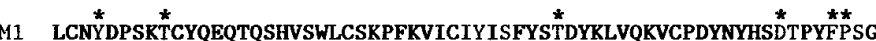
$\mathrm{R}$ LCNYDPSKTCYQEQTQSHVSWLCSKPFKVICIYISFYSTDYKLVQKVCPDYNYHSDTPYFPSC M2 LCNFDPSKICYOEOTOSHVSWLCSKPFKVICIHII FYSVDYKL VOKVCPDYNYHSERPYLSFG
Figure 3. Sequences of neurexophilin genes: implications for evolution. The nucleotide sequences of the $3^{\prime}$ ends of the intron and the large exon of the murine neurexophilin 1 and neurexophilin 2 genes are shown in $A$ and $B$, together with the translated amino acid sequences. Underlined sequences correspond to the intron acceptor site. In $C$, the amino acid sequences of the murine neurexophilin 1 and 2 genes (labeled M1 and M2 on the left) are aligned with the corresponding sequences from rat and bovine neurexophilin (identified as $R$ and $B$ on the left). Residues that are identical in all four sequences are shown in bold typeface. Positions at which the murine neurexophilin 1 gene sequence is identical with the rat neurexophilin sequence and the murine neurexophilin 2 sequence is identical with the bovine neurexophilin sequence, but at which these two groups differ from each other, are marked by an asterisk above the corresponding residue to highlight the close relation of the neurexophilin 1 gene with rat neurexophilin and of the neurexophilin 2 gene with bovine neurexophilin. Overall, the mouse neurexophilin 1 sequence is $61 \%$ identical with the mouse neurexophilin 2 sequence, $99 \%$ identical with the rat neurexophilin sequence, and $63 \%$ identical with the bovine neurexophilin sequence. Conversely, the mouse neurexophilin 2 sequence is $60 \%$ identical with the rat neurexophilin sequence but $88 \%$ identical with the bovine neurexophilin sequence, whereas the bovine and rat neurexophilin sequences are $60 \%$ identical in the region shown. All sequences are numbered on the right. 
with the rat sequence ( 1 mismatch over 80 residues) but exhibits little similarity to bovine or mouse neurexophilin 2 (60 mismatches over 80 residues). Conversely, the $\mathrm{N}$-terminal domain of mouse neurexophilin 2 is $70 \%$ identical with that of bovine neurexophilin but exhibits only $25 \%$ identity with rat or mouse neurexophilin 1 (Fig. 3C). Thus, the murine genome contains at least two neurexophilin genes, one of which corresponds to neurexophilin cDNAs isolated from rat brain and the other corresponds to cDNAs from bovine brain.

\section{Only the neurexophilin 1 gene generates a detectable mRNA in mice}

The genomic cloning results raised two alternative hypotheses: (1) two neurexophilin isoforms are expressed in mice and other mammals from two distinct genes; and (2) mammals primarily express only one neurexophilin isoform, although two genes are present, but different mammals (i.e., rodents vs cattle) may express different isoforms. To differentiate between these two hypotheses, we used RNA blots and in situ hybridization experiments and investigated the expression of neurexophilins in adult mice, rats, and cattle. Of all tissues tested, hybridizing neurexophilin mRNAs were observed only in brain (see below and data not shown). Blots of adult brain RNAs with probes from the two types of neurexophilin surprisingly revealed that in mouse and rat brain, only mRNAs corresponding to the neurexophilin 1 gene (i.e., rat neurexophilin) were detectable (Fig. 4 and data not shown). Conversely, in bovine brain only the bovine form of neurexophilin corresponding to mouse neurexophilin 2 was found (data not shown). Using redundant oligonucleotides hybridizing to conserved sequences in both neurexophilins, polymerase chain reactions were performed with total cDNA from adult mouse brains. Only a single band was amplified that on sequencing was found to contain only neurexophilin 1 (data not shown). Together these data indicate that only transcripts corresponding to neurexophilin 1 are present at measurable levels in adult rat and mouse brain, whereas bovine brain primarily expresses transcripts corresponding to neurexophilin 2. Although we cloned both genes only from mice and not from rats and cows, it seems likely that both genes are also present in these organisms. The presence of distinct neurexophilin genes and their expression pattern in mice, rats, and cattle suggest that neurexophilin genes duplicated in evolution before the divergence of rodents and cattle, and that after the divergence, high expression of one gene was maintained in rodents and of the other gene in cattle.

\section{Pattern of neurexophilin 1 expression in rat brain}

RNA blots indicated that neurexophilin mRNA was detectable only in nervous tissues (data not shown). To examine which cells in brain express neurexophilin, in situ hybridizations were performed with ${ }^{35} \mathrm{~S}$-labeled oligonucleotides from two different regions of the cDNA. All hybridizations were performed in the presence and absence of a 100-fold excess of unlabeled oligonucleotide to control for nonspecific labeling. Hybridization of horizontal rat brain sections revealed expression of rat neurexophilin in neurons in most brain structures in a nonuniform, granular pattern (Fig. 5). Particularly high expression was observed in selected thalamic nuclei (arrowheads) and in the glomerular layer of the olfactory bulb (arrows). The nonuniform labeling pattern was especially evident in the cerebral cortex, which has a peppered appearance (open arrows). Parallel experiments with an oligonucleotide corresponding to

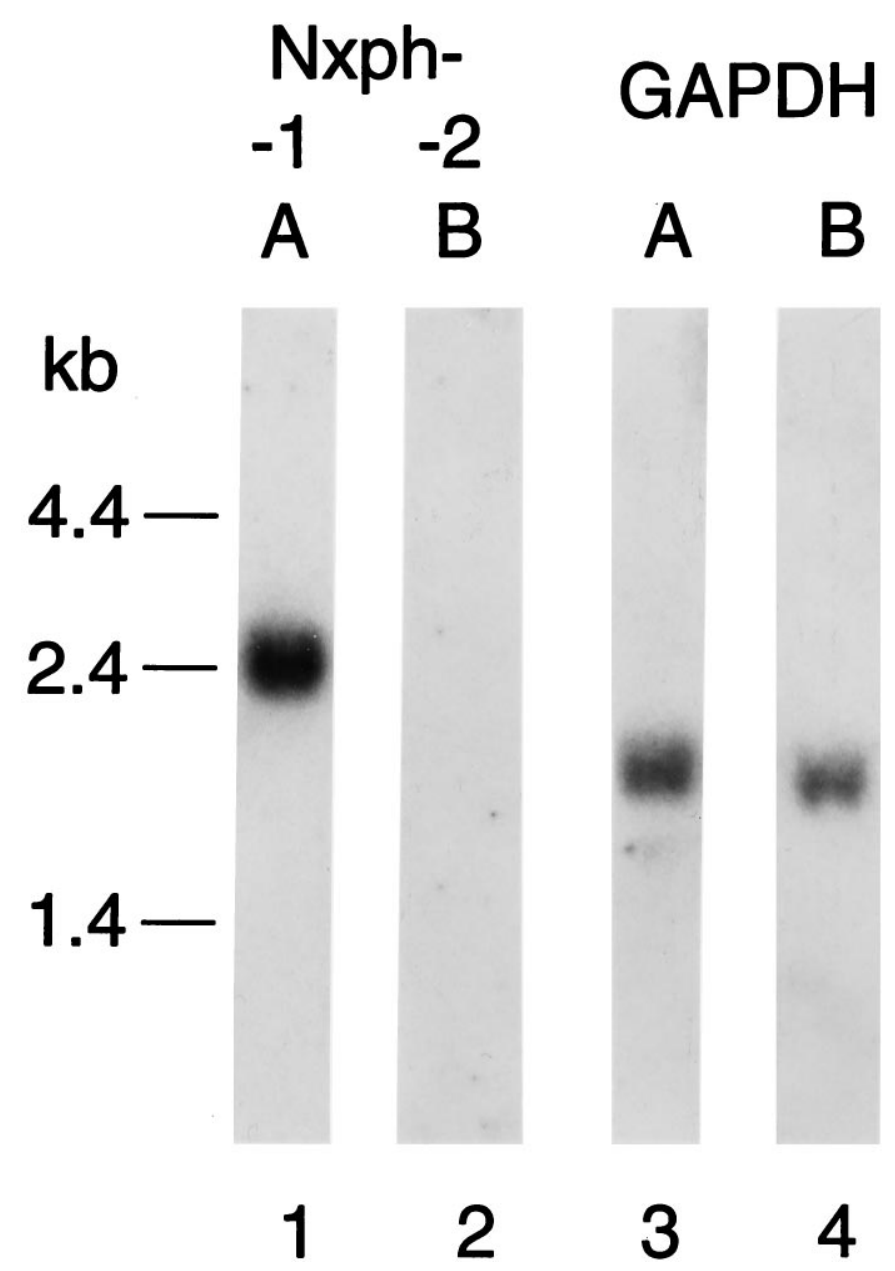

Figure 4. RNA blot analysis of the expression of the two murine neurexophilin genes in mouse brain. Blots containing electrophoretic separated mouse brain RNA were hybridized consecutively with probes from the coding regions of the murine neurexophilin 1 and 2 genes (lanes 1 and 2) and with a GAPDH probe at a positive control (lanes 3 and 4 ). Numbers on the left indicate positions of molecular weight markers in kilobases $(\mathrm{kb})$.

neurexophilin 2 gave no specific hybridization signals in rat brain (data not shown).

The granular pattern of the hybridization signal suggests that only subpopulations of neurons express neurexophilin. To test this hypothesis, expression was examined at the cellular level. A comparison of the expression of synaptotagmin I (Fig. 6A), neurexophilin $(B)$, and neurexins $(C$; the oligonucleotide that was used hybridizes with all neurexin mRNAs) in the hippocampus showed that only a small subpopulation of neurons express high levels of neurexophilin mRNA. Although synaptotagmin I and neurexins are expressed in all identifiable neurons, neurexophilin was not detected in the most abundant classes of neurons in the hippocampal formation, such as the pyramidal cells of the CA1-CA4 regions and the granule cells of the dentate gyrus. Only interspersed cells likely to be inhibitory interneurons contained high levels of neurexophilin mRNA. Similar results were obtained with the cerebellum where again the majority of the mRNA was localized to basket and Golgi cells and not to the predominant granule cells (data not shown). Finally, investigation of the olfactory bulb revealed strong staining of periglomerular neurons (Fig. 7) that probably accounts for the strong signal seen in the olfactory bulb in the overview (Fig. 5). In addition, scattered cells that 
A

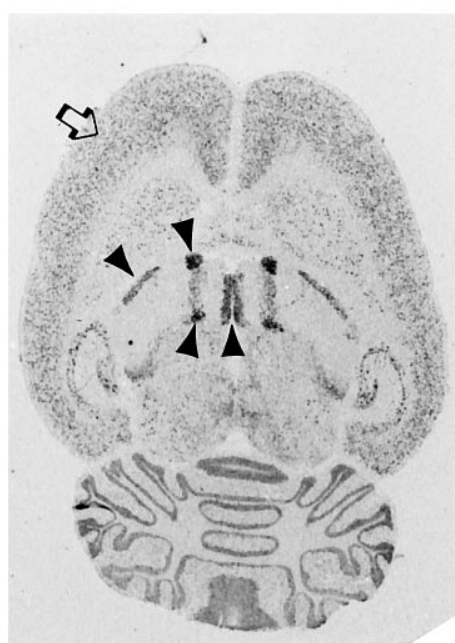

B

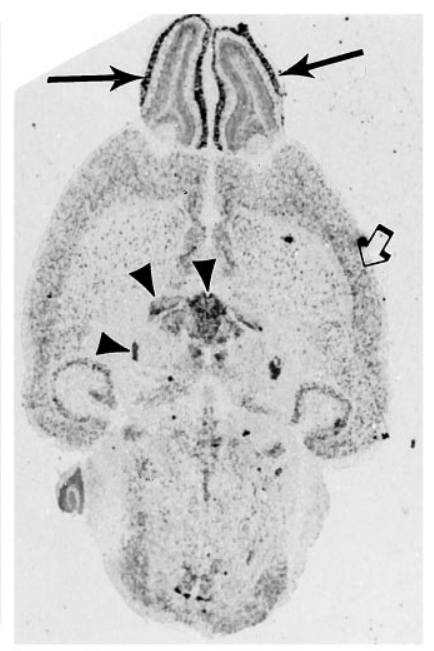

Figure 5. Expression of neurexophilin in rat brain visualized by in situ hybridization. Horizontal sections at two different levels were hybridized with a ${ }^{35}$ S-labeled oligonucleotide specific for neurexophilin and exposed to film without screen. Note the granular hybridization pattern in most brain regions, particularly in the cerebral cortex (open arrows in $A$ and $B$ ). A strong uniform signal is observed only in the periglomerular zone of the olfactory bulb (arrows in $B$ ) and in some of the thalamic nuclei (arrowheads in $A$ and $B$ ), especially the anteroventricular, medial habenular, paraventricular, subgeniculate, reuniens, and reticular thalamic nuclei.

may correspond to tufted cells in the external plexiform layer were also labeled. In contrast, the mitral cells and granule cells, the major cell types in the olfactory bulb, did not express neurexophilin. Together these data suggest that neurexophilin is expressed selectively in subpopulations of neurons, probably primarily inhibitory interneurons.

\section{Neurexophilin co-purifies with bovine and rat neurexin l $\alpha$ on immobilized $\alpha$-latrotoxin}

To test whether the neurexophilins we cloned correspond to the $29 \mathrm{kDa}$ protein that co-purifies with neurexin $\mathrm{I} \alpha$ on immobilized $\alpha$-latrotoxin, we raised antibodies against peptides containing residues $167-179$ of bovine neurexophilin and residues $261-271$ of rat neurexophilin. Testing of these antibodies with rat and bovine neurexophilin expressed in COS cells confirmed that each reacted specifically with the corresponding protein (data not shown). As expected from the limited sequence similarity between rat neurexophilin 1 and bovine neurexophilin 2 in the peptides used for immunizations, the bovine neurexophilin antibody did not react with rat neurexophilin, and the rat neurexophilin antibody recognized bovine neurexophilins only weakly.

We then isolated rat and bovine neurexin I $\alpha$ and associated 29 $\mathrm{kDa}$ proteins by affinity chromatography on immobilized $\alpha$-latrotoxin and analyzed them by immunoblotting for the presence of neurexophilins. The $29 \mathrm{kDa}$ proteins from both species reacted strongly with the neurexophilin antibodies (Fig. 8). This result confirms that the proteins we cloned are identical with the $29 \mathrm{kDa}$ proteins that co-purify with neurexin $\mathrm{I} \alpha$ on immobilized $\alpha$-latrotoxin. Rat neurexophilin isolated on immobilized $\alpha$-latrotoxin reacted only with the anti-rat neurexophilin 1 antibody, and bovine neurexophilin reacted strongly with the bovine neurexophilin 2 antibody and only weakly with the rat neurexophilin 1 antibody. The lack of immunoreactivity of antibodies to bovine neurexophilin 2 with the $\alpha$-latrotoxin affinity eluate from rat brain despite its strong reactivity with the eluate from bovine brain provides further support for the conclusion that neurexophilin 2 is not expressed in rodent brain.

\section{Association of neurexophilins with neurexin I $\alpha$}

Previous studies demonstrated that after purification on immobilized $\alpha$-latrotoxin, the $29 \mathrm{kDa}$ protein corresponding to neurexophilin co-purified with neurexin I $\alpha$ during additional sucrose gradient centrifugation and anion exchange chromatography procedures, suggesting that they are complexed to each other (Petrenko et al., 1993). Because neurexophilin migrates as a monomer on SDS-PAGE in the absence or presence of reducing agents (Fig. 8), neurexin $\mathrm{I} \alpha$ and neurexophilin are not linked to each other by disulfide bonds. To characterize their association better, we probed for conditions under which neurexin $\mathrm{I} \alpha$ and neurexophilin dissociate by performing sucrose gradient centrifugation and gel filtration experiments under denaturing conditions.

Dissociation could not be obtained in $2 \mathrm{M} \mathrm{NaCl}$ on sucrose gradients or in $6 \mathrm{~m}$ urea on gel filtration columns (Fig. 9 and data not shown). Neurexophilin was dissociated only from neurexin I $\alpha$ in $7 \mathrm{~m}$ guanidinium- $\mathrm{HCl}$ at $\mathrm{pH} 1.7$ (Fig. 9). These results suggest that the neurexophilin-neurexin I $\alpha$ complex, although noncovalent, requires complete denaturation for dissociation.

\section{Post-translational processing of neurexophilins}

Neurexophilins co-purified with neurexin I $\alpha$ on immobilized $\alpha$-latrotoxin migrate as $29 \mathrm{kDa}$ proteins on SDS-PAGE (Fig. 8). Digestion with PNGase F (which cleaves N-linked sugars) shifts the apparent size of neurexophilin to $19 \mathrm{kDa}$ (Fig. 10). This result demonstrates that neurexophilins are highly $\mathrm{N}$-glycosylated, a hypothesis that was confirmed with transfected COS cells in which at least three $\mathrm{N}$-glycosylated intermediates of neurexophilin could be distinguished (data not shown).

Although neurexophilin purified on immobilized $\alpha$-latrotoxin migrates at $\sim 19 \mathrm{kDa}$ after deglycosylation, its predicted size after signal sequence cleavage amounts to $\sim 28 \mathrm{kDa}$, suggesting that neurexophilin may be proteolytically processed after synthesis. This raises two possibilities: (1) neurexophilin is a preproprotein that is proteolytically processed in the secretory pathway; and (2) nonspecific proteolysis during purification leads to partial degradation. The simplest method of distinguishing between these hypotheses would be to determine the size of neurexophilin in fresh total brain homogenates before any nonspecific proteolysis can occur; however, we were unable to detect neurexophilin in total brain homogenates, possibly because of the relatively low affinity of our neurexophilin antibodies or of the low abundance of the protein, making it currently impossible to differentiate between these alternatives.

\section{Binding of recombinant neurexophilin to the extracellular domain of neurexin $\mathbf{l} \alpha$}

The fact that neurexophilins are N-glycosylated, contain a signal peptide, and lack a transmembrane region suggests that they are secreted and presumably bind to the extracellular part of neurexin $\mathrm{I} \alpha$. To test this directly, the extracellular domains of neurexin I $\alpha$ were produced as a recombinant fusion protein with the $\mathrm{Fc}$ portion of human IgG. The neurexin-IgG fusion protein as well as a control IgG Fc domain were immobilized on protein A-agarose and mixed with neurexophilin produced as a recombinant GSTfusion protein in bacteria. Quantitative binding of neurexophilin was observed only to neurexin I $\alpha$ but not to the control (Fig. 11), suggesting that neurexophilin binds to the extracellular domains of neurexin $\mathrm{I} \alpha$. 
A

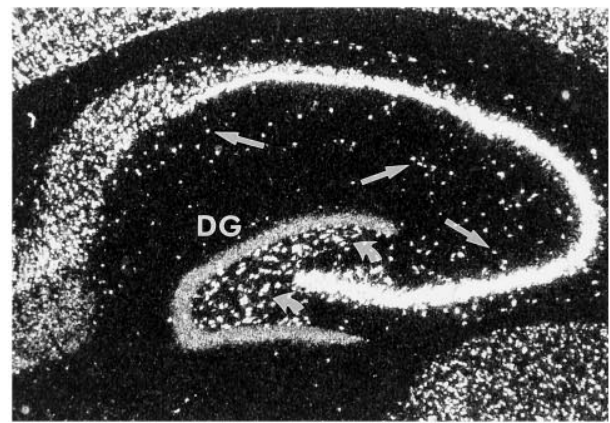

B

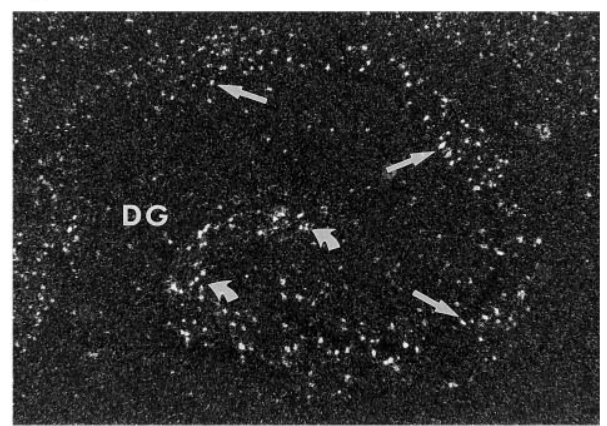

C

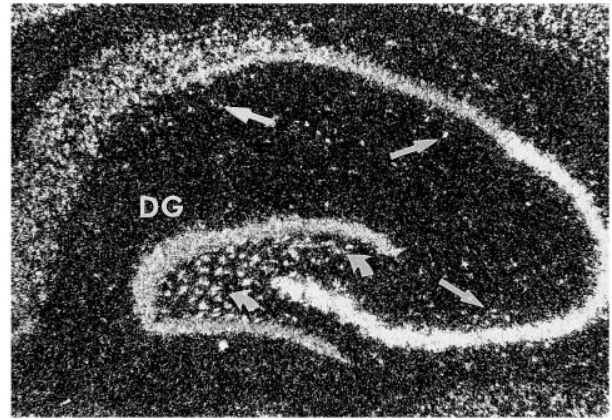

Figure 6. Distribution of neurexophilin mRNA in hippocampus. Rat hippocampal sections were hybridized with ${ }^{35}$ S-labeled oligonucleotides corresponding to synaptotagmin I $(A)$ or neurexophilin $(B)$ or to a consensus sequence present in all neurexins $(C)$. Note the presence of neurexins and synaptotagmin I in apparently all neurons in the hippocampus, whereas neurexophilin is absent from most hippocampal neurons, including pyramidal cells of the hippocampus proper and granule cells of the dentate gyrus $(D G)$; however, neurexophilin is present at high levels in scattered neurons of the hippocampus (straight arrows) and dentate gyrus (curved arrows) that seem to be primarily inhibitory interneurons.

\section{DISCUSSION}

Neurexins constitute a polymorphic family of neuronal cell surface proteins (Ushkaryov et al., 1992, 1994; Ullrich et al., 1995). Neurexin $\mathrm{I} \alpha$, the founding member of the family, was discovered in studies on the mechanism of action of $\alpha$-latrotoxin, a potent excitatory neurotoxin. Affinity chromatography of rat and bovine brain proteins on immobilized $\alpha$-latrotoxin revealed that two proteins were purified specifically in a $\mathrm{Ca}^{2+}$-dependent manner (Petrenko et al., 1993): a set of high molecular weight proteins that represent splice variants of neurexin $\mathrm{I} \alpha$, and a low molecular weight protein that we have now named neurexophilin. The two proteins co-purify with each other in several separation procedures, suggesting that they are present in a complex; however, recombinant neurexin $\mathrm{I} \alpha$ binds $\alpha$-latrotoxin with high affinity and does not need to be complexed to neurexophilin for binding (Davletov et al., 1995), indicating that neurexophilin is purified on immobilized $\alpha$-latrotoxin piggy-back in a complex with neurexin I $\alpha$. We have now studied the molecular nature of neurexophilin and of its association with neurexin $\mathrm{I} \alpha$.

We have cloned cDNAs encoding neurexophilins from rat and bovine brain, isolated genomic clones encoding most of the coding regions for two neurexophilins from mice, investigated their pattern of expression, and studied the biochemical properties of neurexophilin and its binding to neurexin $\mathrm{I} \alpha$. The structures of rat and bovine neurexophilins revealed that they are novel proteins without significant similarity to current databank entries. Sequence analyses suggested a four-domain structure for neurexophilins: (1) an N-terminal signal peptide; (2) an N-terminal nonconserved sequence; (3) a conserved region containing no cysteine residues but three potential $\mathrm{N}$-glycosylation sites; and (4) a C-terminal domain characterized by six conserved cysteine residues (Fig. 1). No hydrophobic region except for the putative signal peptide was observed. The presence of a signal sequence in neurexophilin and the absence of hydrophobic transmembrane regions suggest that neurexophilin is a secreted protein. This hypothesis was supported by the demonstration that neurexophilin is highly $\mathrm{N}$-glycosylated and has to transverse the secretory pathway.

Neurexophilin was purified first in a complex with neurexin I $\alpha$ (Petrenko et al., 1993). Studies on the nature of this complex
A

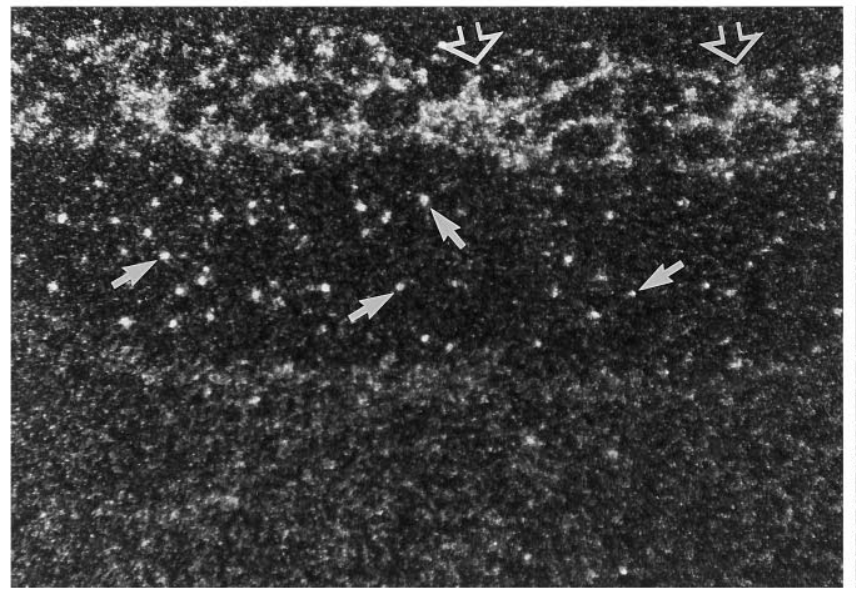

B

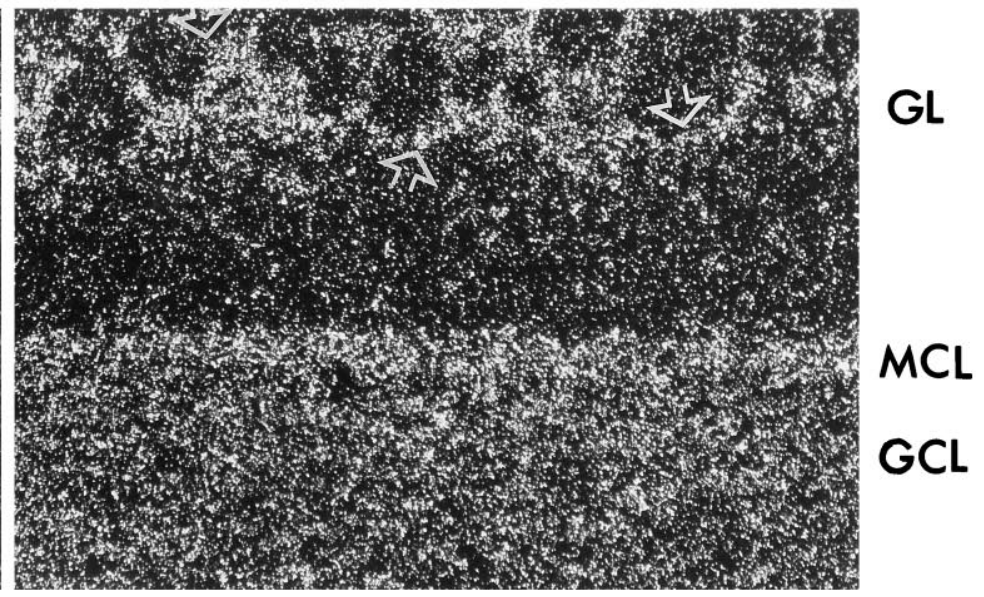

Figure 7. Distribution of neurexophilin mRNA in the olfactory bulb. Panels show sections of rat olfactory bulb hybridized with ${ }^{35}$ S-labeled oligonucleotides specific for neurexophilin $(A)$ or for neurexin $\mathrm{I} \alpha(B)$. Neurexophilin mRNA is present at high levels in two cell types: periglomerular neurons in the glomerular layer $(G L)$ that are uniformly positive for neurexophilin mRNA (open arrowheads) and scattered neurons of the external plexiform layer that may correspond to tufted cells (full arrows). Note the absence of neurexophilin mRNA from the mitral cell layer $(M C L)$ and the granule cell layer $(G C L)$, which are strongly positive for neurexin $\mathrm{I} \alpha$. 


\begin{tabular}{|r|c|c|c|c|c|c|c|c|}
\hline Rat & + & - & + & - & + & - & + & - \\
\hline Bovine & - & + & - & + & - & + & - & + \\
\hline$\beta-M E$ & - & - & + & + & - & - & + & + \\
\hline
\end{tabular}

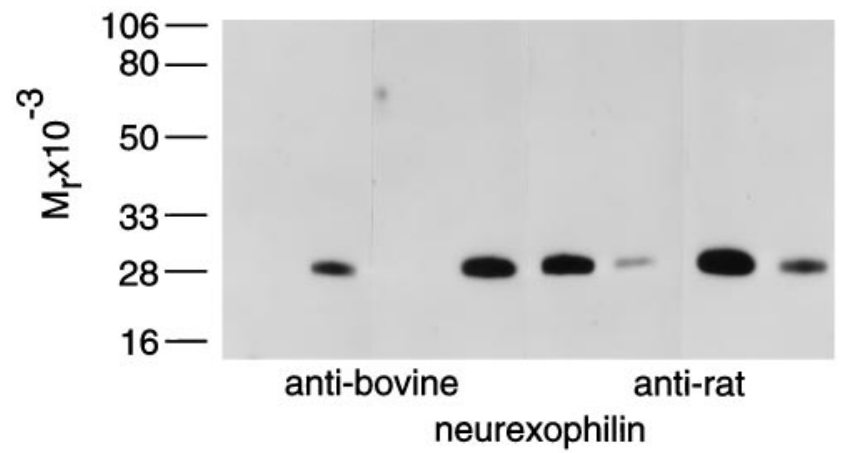

Figure 8. Neurexophilins are co-purified with neurexin $\mathrm{I} \alpha$ by affinity chromatography on immobilized $\alpha$-latrotoxin from rat and bovine brain. Rat and bovine $\alpha$-latrotoxin binding proteins were purified from brain homogenates by affinity chromatography on immobilized $\alpha$-latrotoxin and analyzed by SDS-PAGE in the presence or absence of $\beta$-mercaptoethanol $(\beta-M E)$. Gels were immunoblotted with antibodies against synthetic peptides corresponding to residues 167-179 of bovine neurexophilin (left) or to residues 261-271 of rat neurexophilin (right). Numbers on the left indicate positions of molecular weight markers.

revealed an unexpectedly tight association (Fig. 9). Only strong denaturants (SDS, guanidinium hydrochloride) could dissociate them from each other. Comparison of the size of the deglycosylated peptide backbone of purified neurexophilin complexed to neurexin $\mathrm{I} \alpha$ with the calculated size of the protein after signal sequence cleavage revealed that the purified protein is smaller than expected. It is unclear at present whether this size discrepancy is attributable to proteolytic processing of neurexophilin during biosynthesis or whether the protein is partially degraded during purification by nonspecific proteases. The low affinity of our antibodies and the low abundance of neurexophilin did not allow us to address this question directly. Antibodies against the $\mathrm{C}$ terminus of neurexophilin, however, react with the purified protein complexed to neurexin $\mathrm{I} \alpha$, suggesting that it contains the $\mathrm{C}$ terminus and lacks the nonconserved $\mathrm{N}$ terminus. Because in many secreted proteins that are processed from precursor proteins (such as NGF) the removed propeptide often is not evolutionarily conserved, this observation supports the notion that neurexophilin is synthesized as a prepropeptide and proteolytically processed in the secretory pathway. Future experiments will have to address this question.

In situ hybridizations demonstrated that neurexophilin is expressed in a restricted pattern strikingly different from that of neurexins. Neurexins are present in all identifiable neurons, with distinct heterogeneous expression patterns for different subtypes (Ullrich et al., 1995). By contrast, no neurexophilin mRNA was detected in the majority of neurons. Only a relatively small subclass of neurons that corresponded primarily to inhibitory interneurons contained abundant levels of neurexophilin mRNA. These data suggest that neurexophilin, unlike neurexins, is not expressed universally in neurons.

The overall sequence homology between bovine and rat neurexophilin is surprisingly low. There is little similarity between the bovine and rat proteins in the putative signal sequence and the $\mathrm{N}$-terminal 80 residues that follow the signal peptide. Even in the conserved $\mathrm{C}$-terminal domains, the sequence conservation is much lower than that observed in comparisons between rat and bovine neurexins ( $>95 \%$ identity as opposed to $85 \%$ identity in neurexophilins; Ullrich et al., 1995). This observation prompted us to investigate the possibility that the rat and bovine cDNAs we cloned are not exact homologs but are isoforms.

Isolation of genomic clones encoding neurexophilins from mice revealed the presence of two murine neurexophilin genes, referred to as the neurexophilin 1 and 2 genes. The similarity between the structures and sequences of the two murine neurexophilin genes suggests that they resulted from an evolutionary gene duplication event that was followed by sequence divergence with selective conservation of the C-terminal sequences of neur-

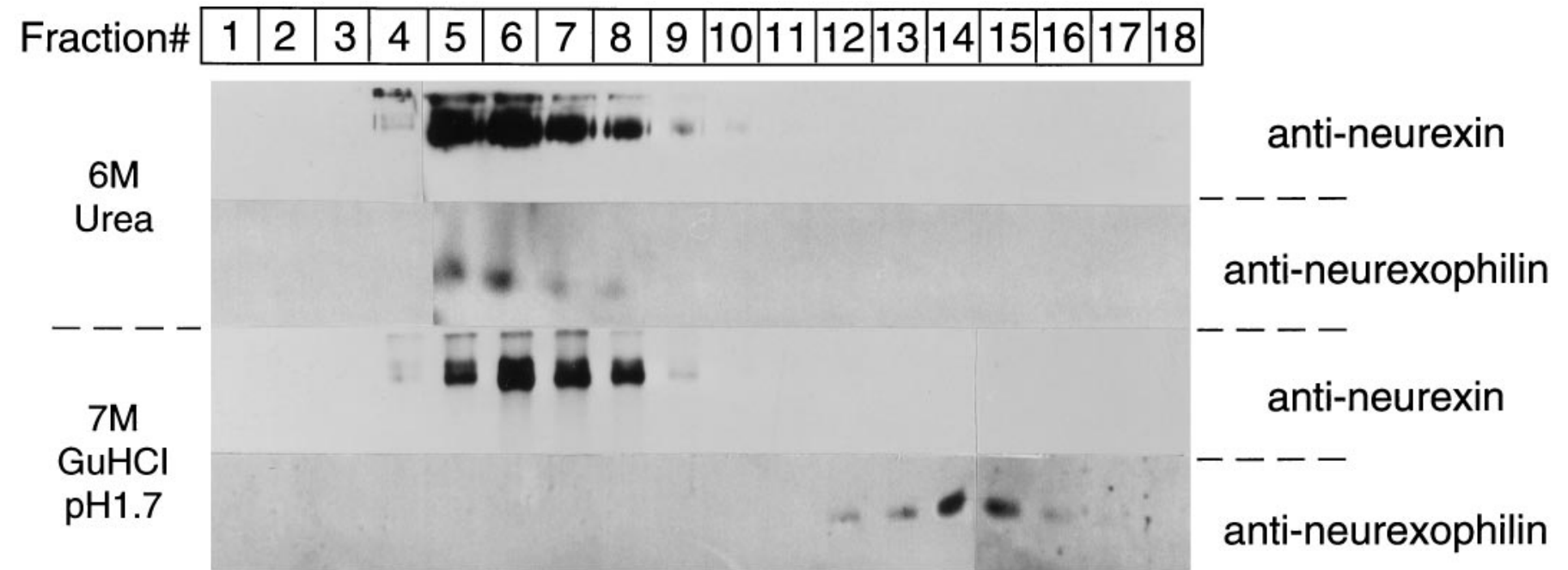

Figure 9. Tight binding of neurexophilin to neurexins. The stability of the neurexin-neurexophilin complex purified on immobilized $\alpha$-latrotoxin was analyzed by gel filtration on a Sephacryl S-300 column in $6 \mathrm{~m}$ urea, $75 \mathrm{~mm}$ Tris- $\mathrm{HCl}, \mathrm{pH} 7.4$ (top), or in $7 \mathrm{~m}$ guanidinium- $\mathrm{HCl}$, pH 1.7 (bottom). The first $1.8 \mathrm{ml}$ of column eluate was discarded, and $0.1 \mathrm{ml}$ fractions were subjected to immunoblotting using antibodies against the C terminus of neurexins or bovine neurexophilin, as indicated. Immunoreactive bands were visualized by ECL. Note that neurexophilin comigrates with neurexin I $\alpha$ at high molecular weights in $6 \mathrm{M}$ urea but dissociates from neurexin I $\alpha$ in $7 \mathrm{M}$ guanidinium- $\mathrm{HCl}$. In the samples containing urea, protein migration in the gel is slightly distorted. 


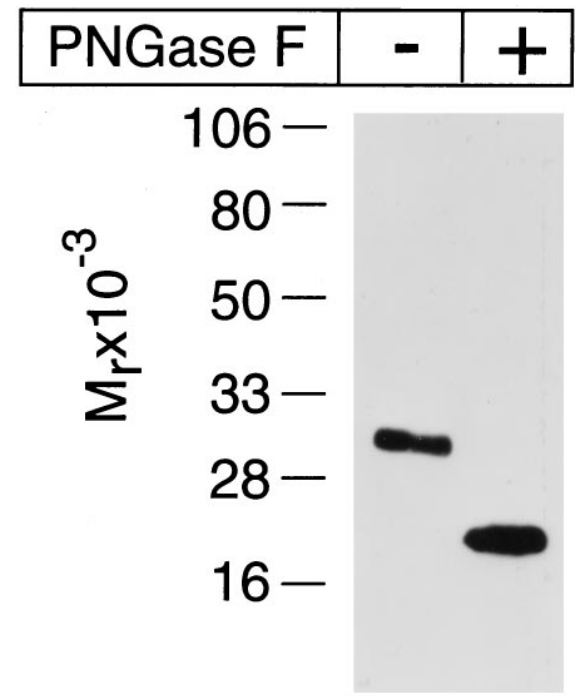

Figure 10. N-glycosylation of neurexophilin. Neurexophilin complexed to neurexin $\mathrm{I} \alpha$ was purified by affinity chromatography on immobilized $\alpha$-latrotoxin and incubated with or without PNGase F. Samples $(0.5 \mu \mathrm{g}$ protein) were analyzed by immunoblotting with antibodies to neurexophilin. Numbers on the right indicate positions of molecular weight markers.

exophilins. The following evidence supports the hypothesis that the two neurexophilin genes do not encode two isoforms coexpressed in the same organism, but rather one or the other neurexophilin gene is expressed selectively at high levels in different species. (1) Multiple cDNAs isolated from rat and bovine brain libraries under low stringency conditions exclusively encoded only one type of neurexophilin that corresponded to either neurexophilin 1 (rat) or neurexophilin 2 (bovine). (2) No neurex-

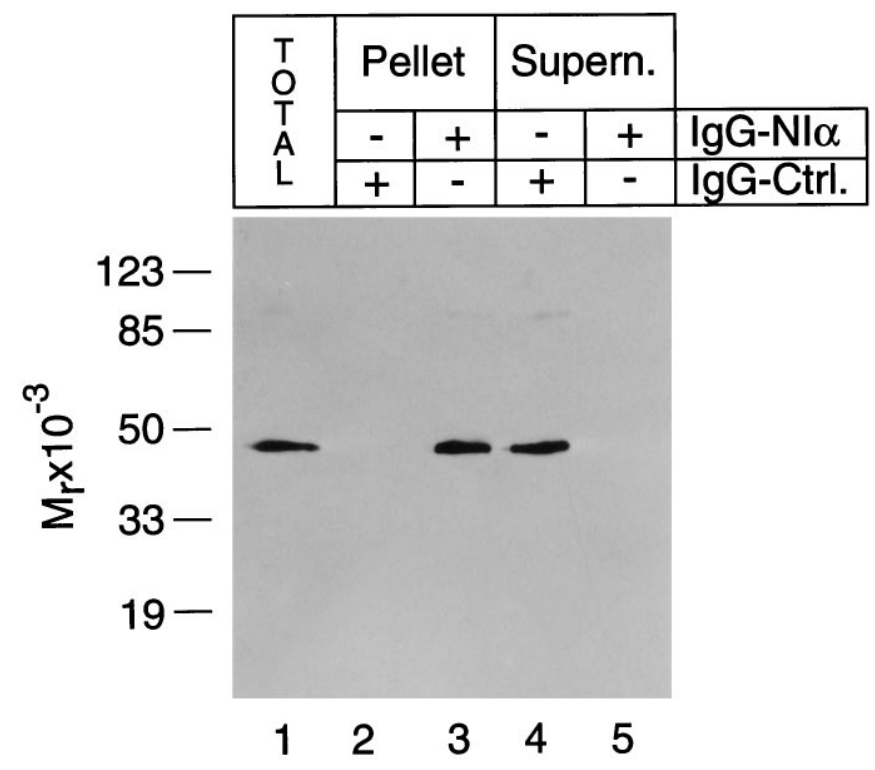

Figure 11. Binding of recombinant neurexophilin to neurexin $\operatorname{I} \alpha-\operatorname{IgG}$ fusion protein and to IgG control protein. Purified GST-neurexophilin fusion protein $(0.1 \mu \mathrm{g}$, lane 1$)$ was mixed with immobilized control IgG fusion protein (lanes 2 and 4 ) or with neurexin $\operatorname{I} \alpha$-IgG fusion protein (lanes 3 and 5), incubated, and centrifuged. Pellets and supernatants were analyzed by immunoblotting for neurexophilin, demonstrating retention of neurexophilin with the control $\operatorname{IgG}$ matrix but pelleting with the neurexin $\mathrm{I} \alpha$-IgG matrix. ophilin 2 mRNA could be detected in RNA blots in rat and murine brain RNA but was expressed abundantly in bovine brain RNA, whereas neurexophilin 1 mRNA was abundant in rat and mouse RNA. (3) No neurexophilin 2 sequence could be amplified from mouse cDNA by the polymerase chain reaction. (4) In situ hybridizations failed to detect cells expressing neurexophilin 2 in adult rat brain. (5) Antibodies to neurexophilins 1 and 2 failed to detect neurexophilin 2 in rat $\alpha$-latrotoxin affinity eluate. Although these data do not completely rule out expression of neurexophilin 2 in rodents or of neurexophilin 1 in cattle, especially in tissues and at developmental time points distinct from those studied here, they are compatible with the hypothesis that at some time in evolution, after the neurexophilin genes had duplicated and diverged, expression of the neurexophilin 2 gene was depressed in rodents, and expression of the neurexophilin 1 gene was depressed in cattle. This resulted in the expression of distinct types of genes in rodents and cattle.

Together our findings characterize a novel neuronal glycoprotein that has an unusual evolutionary history, is expressed in a small subset of neurons, and binds to neurexin $\mathrm{I} \alpha$ and potentially other neurexins. Future studies will have to determine whether neurexophilin serves as a ligand for neurexins and what the determinants and functional implications of this interaction are, especially in view of their unusual relative distributions in brain.

\section{REFERENCES}

Davletov B, Krasnoperov V, Hata Y, Petrenko AG, Südhof TC (1995) High affinity binding of $\alpha$-latrotoxin to recombinant neurexin I $\alpha$. J Biol Chem 270:23903-23905.

Frontali N, Ceccarelli B, Gorio A, Mauro A, Siekevitz P, Tzeng MC, Hurlbut WP (1976) Purification from black widow spider venom of a protein factor causing the depletion of synaptic vesicles at neuromuscular junctions. J Cell Biol 68:462-479.

Geppert M, Ushkaryov YA, Hata Y, Davletov B, Petrenko AG, Südhof TC (1992) Neurexins. Cold Spring Harbor Symp Quant Biol LVII: 483-490.

Gerfen CR, Young WS, Emson P (1992) In situ hybridization histochemistry with oligonucleotides for localization of messenger RNA in neurones. In: Experimental neuroanatomy: a practical approach (Bolam JP, ed), pp 173-186. Oxford: IRL.

Gorman C (1985) High efficiency gene transfer into mammalian cells. In: DNA cloning, Vol II (Glover DM, ed), pp 143-190. Oxford: IRL.

Grasso A, Alema S, Rufini S, Senni MI (1980) Black widow spider toxin-induced calcium fluxes and transmitter release in a neurosecretory cell line. Nature 283:774-776.

Hata Y, Butz S, Südhof TC (1996) CASK: a novel $d l g /$ PSD95 homolog with an N-terminal calmodulin-dependent protein kinase domain identified by interaction with neurexins. J Neurosci 16:2488-2494.

Hata Y, Davletov B, Petrenko R, Jahn R, Südhof TC (1993) Interaction of synaptotagmin with the cytoplasmic domains of neurexins. Neuron 10:307-315.

Ichtchenko K, Hata Y, Nguyen T, Ullrich B, Missler M, Moomaw C, Südhof TC (1995) Neuroligin 1: a splice-site specific ligand for $\beta$-neurexins. Cell 81:435-443.

Ichtchenko K, Nguyen T, Südhof TC (1996) Structure, alternative splicing, and neurexin binding of multiple neuroligins. J Biol Chem 271:2676-2682.

Johnston PA, Jahn R, Südhof TC (1989) Transmembrane topography and evolutionary conservation of synaptophysin. J Biol Chem 264:1268-1273.

McMahon HT, Rosenthal L, Meldolesi J, Nichols DG (1990) $\alpha$-Latrotoxin releases both vesicular and cytoplasmic glutamate from isolated nerve terminals. J Neurochem 55:2039-2047.

Misler S, Hurlbut WP (1979) Action of black widow spider venom on quantized release of acetylcholine at the frog neuromuscular junction: dependence upon external Mg2+. Proc Natl Acad Sci USA 76:991-995.

Petrenko AG, Kovalenko VA, Shamotienko OG, Surkova IN, Tarasyuk TA, Ushkaryov YA, Grishin EV (1990) Isolation and properties of the alpha-latrotoxin receptor. EMBO J 9:2023-2027. 
Petrenko AG, Lazaryeva VD, Geppert M, Tarasyuk TA, Moomaw C, Khokhlatchev AV, Ushkaryov YA, Slaughter C, Nasimov IV, Südhof TC (1993) Polypeptide composition of the $\alpha$-latrotoxin receptor. J Biol Chem 268:1860-1867.

Petrenko AG, Perin MS, Davletov BA, Ushkaryov YA, Geppert M, Südhof TC (1991) Binding of synaptotagmin to the $\alpha$-latrotoxin receptor implicates both in synaptic vesicle exocytosis. Nature 353:65-68.

Rosenthal L, Meldolesi J (1989) Alpha-latrotoxin and related toxins. Pharmacol Ther 42:115-134.

Sambrook J, Fritsch EF, Maniatis T (1989) Molecular cloning: a laboratory manual, 2nd ed. Cold Spring Harbor, NY: Cold Spring Harbor Laboratory.

Sanger F, Nicklen S, Coulson AR (1977) DNA sequencing with chainterminating inhibitors. Proc Natl Acad Sci USA 74:5463-5467.

Smith DB, Johnson KS (1988) Single-step purification of polypeptides expressed in Escherichia coli as fusions with glutathione $S$-transferase. Gene 67:31-40.

Südhof TC (1990) The structure of the human synapsin I gene and protein. J Biol Chem 265:7849-7852.

Tzeng MC, Siekevitz P (1979) The binding interaction between alpha- latrotoxin from black widow spider venom and a dog cerebral cortex synaptosomal membrane preparation. J Neurochem 33:263-274.

Ullrich B, Ushkaryov YA, Südhof TC (1995) Cartography of neurexins: more than 1000 isoforms generated by alternative splicing and expressed in distinct subsets of neurons. Neuron 14:497-507.

Ushkaryov YA, Südhof TC (1993) Neurexin III $\alpha$ : extensive alternative splicing generates membrane-bound and soluble forms in a novel neurexin. Proc Natl Acad Sci USA 90:6410-6414.

Ushkaryov YA, Hata Y, Ichtchenko K, Moomaw C, Afendis S, Slaughter CA, Südhof TC (1994) Conserved domain structure of $\beta$-neurexins. J Biol Chem 269:11987-11992.

Ushkaryov YA, Petrenko AG, Geppert M, Südhof TC (1992) Neurexins: synaptic cell surface proteins related to the $\alpha$-latrotoxin receptor and laminin. Science 257:50-56.

Valtorta F, Maddedu L, Meldolesi J, Ceccarelli B (1984) Specific localization of the alpha-latrotoxin receptor in the nerve terminal plasma membrane. J Cell Biol 99:124-132.

von Heijne G (1987) Sequence analysis in molecular biology: treasure trove or trivial pursuit. San Diego, CA: Academic. 